\title{
Extended-Release Dinalbuphine Sebacate Versus Intravenous Patient-Controlled Analgesia with Fentanyl for Postoperative Moderate-to-Severe Pain: A Randomized Controlled Trial
}

\author{
Tsung-Kun Chang • Ching-Wen Huang • Wei-Chih Su • \\ Hsiang-Lin Tsai · Cheng-Jen Ma • Yung-Sung Yeh • Yen-Cheng Chen • \\ Ching-Chun Li $\cdot$ Kuang-I. Cheng $\cdot$ Miao-Pei Su $\cdot$ Jaw-Yuan Wang (D)
}

Received: August 26, 2020 / Accepted: September 14, 2020 / Published online: September 29, 2020

(C) The Author(s) 2020

\begin{abstract}
Introduction: Post-operative pain control remains unsatisfactory in patients after laparotomy. This study aimed to evaluate the efficacy, safety, and quality of life with a single dose of extended-release dinalbuphine sebacate (ERDS) pre-operatively to intravenous patient-con-
\end{abstract}

Digital Features To view digital features for this article go to https://doi.org/10.6084/m9.figshare.12942845.

T.-K. Chang · C.-W. Huang · W.-C. Su · H.-L. Tsai ·

C.-J. Ma · Y.-S. Yeh · Y.-C. Chen · C.-C. Li .

J.-Y. Wang (ه)

Division of Colorectal Surgery, Department of

Surgery, Kaohsiung Medical University Hospital,

Kaohsiung Medical University, Kaohsiung, Taiwan

e-mail: cy614112@ms14.hinet.net;

jawyuanwang@gmail.com

T.-K. Chang · C.-W. Huang · W.-C. Su · Y.-S. Yeh ·

J.-Y. Wang

Graduate Institute of Clinical Medicine, College of Medicine, Kaohsiung Medical University,

Kaohsiung, Taiwan

C.-W. Huang · H.-L. Tsai · J.-Y. Wang

Department of Surgery, Faculty of Medicine, College of Medicine, Kaohsiung Medical University,

Kaohsiung, Taiwan

C.-J. Ma

Division of General and Digestive Surgery,

Department of Surgery, Kaohsiung Medical

University Hospital, Kaohsiung Medical University,

Kaohsiung, Taiwan trolled analgesia (PCA) with fentanyl in patients undergoing laparotomy.

Methods: This was a prospective, open-label, randomized controlled study. Of 110 randomized patients, 107 completed all assessments. The area under the curve (AUC) of visual analogue scale (VAS) from baseline to $48 \mathrm{~h}$ after surgery, VAS throughout 7 days after surgery, post-operative analgesics use, quality of life, satisfaction, and safety were evaluated.

Y.-S. Yeh

Division of Trauma and Critical Care, Department of Surgery, Kaohsiung Medical University Hospital, Kaohsiung Medical University, Kaohsiung, Taiwan

K.-I. Cheng · M.-P. Su

Department of Anesthesiology, Kaohsiung Medical University Hospital, Kaohsiung Medical University, Kaohsiung, Taiwan

K.-I. Cheng · M.-P. Su

Department of Anesthesiology, Faculty of Medicine, College of Medicine, Kaohsiung Medical University, Kaohsiung, Taiwan

\section{J.-Y. Wang}

Center for Cancer Research, Kaohsiung Medical

University, Kaohsiung, Taiwan

J.-Y. Wang

Clinical Pharmacogenomics and

Pharmacoproteomics, School of Pharmacy, Taipei

Medical University, Taipei, Taiwan 
Results: The AUC of VAS from baseline to $48 \mathrm{~h}$ after surgery were 118.6 [97.5\% confidence interval (CI) 95.6-141.6] in ERDS group and 176.13 (97.5\% CI 150.8-201.4) in PCA group, which showed the non-inferiority because the upper limit of the 97.5\% CIs of ERDS group was lower than the lower limit of PCA group $(P<0.001)$, but also had superiority in favor of ERDS group $(P<0.001)$. ERDS group reported a significant reduction in VAS pain intensity at 4 , $24,32,72,120$, and $144 \mathrm{~h}$ after surgery, and better quality of life $(P<0.05)$. The safety profile was comparable between ERDS and PCA groups.

Conclusions: In patients undergoing laparotomy, a single dose of dinalbuphine sebacate was superior to intravenous PCA with fentanyl on lower pain intensity and better quality of life.

Trial Registration: NCT03296488.

Keywords: Fentanyl; Nalbuphine; PCA; Laparotomy; Post-operative analgesia

\section{Key Summary Points}

Why carry out this study?

Post-operative pain control remains unsatisfactory in patients with colorectal cancer after laparotomy.

Extended-release dinalbuphine sebacate (ERDS) is a prodrug of nalbuphine, a kappa receptor agonist and mu receptor of antagonist, has revealed in effective pain control and less postoperative ketorolac consumption after hemorrhoidectomy.

We hypothesized pre-operative administration of ERDS is non-inferior to intravenous patient-controlled analgesia (IV PCA) with fentanyl in pain intensity.

\section{What was learned from the study?}

This study demonstrated that a single dose of ERDS not only achieved pre-defined non-inferior margin but was also superior to IV PCA with fentanyl in pain intensity and quality of life.
ERDS provided a tolerable safety profile; the most frequent adverse drug reaction was pyrexia. There were no significant differences for any of the safety assessments between the two treatment groups.

\section{DIGITAL FEATURES}

This article is published with digital features to facilitate understanding of the article. To view digital features for this article go to https://doi. org/10.6084/m9.figshare.12942845.

\section{INTRODUCTION}

One-third of patients experienced severe acute pain on the first day after laparotomy and 18\% of them experienced chronic post-surgical pain at 4 years after surgery $[1,2]$. More evidence has reported that inadequate post-operative pain management leads to negative effects on physical function, recovery period, and post-surgical complications [3-5]. Various analgesic methods have recently been developed, despite differences among these approaches, the main target is to achieve post-operative pain relief and improve quality of life. Intravenous patientcontrolled analgesia (IV PCA) has been utilized over decades to relieve post-operative acute pain and has achieved high patient satisfaction. However, many issues still limit its clinical use, such as catheter infiltration, intravenous tube obstruction, and machine programming errors [6].

The mu opioid receptor (MOR) agonists acting on the central nervous system (CNS), such as morphine and fentanyl, are common choices for IV PCA because intravenous administration immediately increases the risk of opioid-related adverse events [7]. Although studies on the use of IV PCA with fentanyl reported effective pain relief and high satisfaction on the first day after surgery, they are still scarce to observe pain management in surgeries that need long-day recovery period, like laparotomy $[8,9]$. Extended-release dinalbuphine sebacate (ERDS, 
Naldebain ${ }^{\circledR}$, Lumosa Therapeutics, Taipei, Taiwan) is a pro-drug of nalbuphine, which acts as a mixed kappa opioid agonist/mu opioid antagonist providing sufficient analgesia for moderate to severe pain after a single intramuscular administration. ERDS can rapidly hydrolyze to nalbuphine by esterases in the human bloodstream [10]. A randomized study has reported that pre-operative intramuscular administration of ERDS exhibits a well-tolerated safety profile and weekly analgesic effect, less post-operative analgesics use, and prolonged time to the first use of analgesics in patients undergoing hemorrhoidectomy [11]. In addition, ERDS contributes a relatively low concentration of nalbuphine acting on the CNS, which served as background analgesia, but also helps to prevent MOR agonist-induced side effects such as pruritus, nausea, and vomiting [12-14].

In this study, which included adult patients undergoing laparotomy, we compared the drug efficacy and safety and the quality of life between patients receiving pre-emptive analgesia with a single dose of ERDS and those receiving post-operative pain management using IV PCA with fentanyl. The primary outcome was to evaluate the AUC of VAS from baseline to $48 \mathrm{~h}$ after surgery between ERDS and IV PCA with fentanyl. In addition, the impact of pre-emptive ERDS and post-operative IV PCA with fentanyl after laparotomy on quality of life will also be evaluated.

\section{METHODS}

This was a prospective, randomized controlled trial that was conducted in accordance with the Declaration of Helsinki, international conference on harmonization guidelines for good clinical practice, and local regulations. The study was approved by the hospital's research ethics committee (Institutional Review Board of Kaohsiung Medical University Hospital, KMUHIRB-F(I)-20170089) and was registered in the Clinicaltrials.gov (NCT03296488, Principal investigator: Wang JY, M.D., Ph.D.). The study protocol was conducted at the Kaohsiung Medical University Hospital from November 2017 to July 2019, in accordance with the
Declaration of Helsinki, International Conference on Harmonization of Good Clinical Practice, and local regulatory requirements. All participants signed informed consent before initiation of study assessments. Patients were enrolled from November 2017 to July 2019 in Kaohsiung Medical University Hospital. Patients aged between 20 and 80 years who were scheduled to undergo elective laparotomy with general anesthesia were eligible if they were considered as class I-III according to the American Society of Anesthesiologists (ASA) physical status classification. Patients with the following criteria were excluded: body mass index (BMI) below $18 \mathrm{~kg} / \mathrm{m}^{2}$ or above $30 \mathrm{~kg} / \mathrm{m}^{2}$; pregnant or lactating patients; history of open laparotomy; allergy, sensitivity or contraindication to local anesthetics, opioids or ingredients of the study medications; history of chronic opioid use; severe comorbidity; and inability to use a PCA device.

All patients were assigned randomly with equal ratio to receive either a single dose of ERDS injection or intravenous PCA with fentanyl according to a computer-generated simple randomization code. All patients were instructed on the visual analogue scale (VAS), BPI, time to use rescue medication, the PCA device (PCA group only), and other assessments included in the study. Anesthesia was induced in both groups with $1.6 \mathrm{mg} / \mathrm{kg}$ propofol, $0.8 \mathrm{mg} / \mathrm{kg}$ lidocaine, $1 \mu \mathrm{g} / \mathrm{kg}$ fentanyl, $0.1 \mathrm{mg} / \mathrm{kg}$ dexamethasone, and $0.8 \mathrm{mg} / \mathrm{kg}$ rocuronium bromide; maintained with 5-10 mg morphine, 50-100 $\mu \mathrm{g}$ fentanyl, and $0.5 \mathrm{mg} / \mathrm{kg}$ rocuronium bromide. For study intervention, in the ERDS group, a single dose of $150 \mathrm{mg}$ dinalbuphine sebacate (75 mg/ml, $2 \mathrm{ml} /$ vial) was injected intramuscularly 1 day before surgery. The PCA group received fentanyl for $48 \mathrm{~h}$ after surgery via intravenous PCA. The PCA device was programmed to give a bolus of $20 \mu \mathrm{g}$ fentanyl ( $4 \mu \mathrm{g}$ / $\mathrm{ml}, 5 \mathrm{ml} /$ dose) with a minimal lockout interval of $20 \mathrm{~min}$ and no background infusion in the PCA group. The recommended setting of fentanyl dosage in the PCA group was $600 \mu \mathrm{g}$ within $48 \mathrm{~h}$ after surgery according to the average fentanyl use in previous laparotomy cases, which was equivalent to the maximum exposure of ERDS in the same period by 
pharmacokinetic simulation. After the patients stayed in the ward, ketorolac and morphine were recommended as supplemental analgesia for both treatment groups and the total amount was documented on a daily base. During the study, both treatment groups were evaluated by daily observation including vital signs, injection site reactions, concomitant medications, and adverse events starting from the administration of the study medications to discharge. VAS $(0-10 \mathrm{~cm} ; 0=$ no pain, $10=$ worst pain $)$ was used to evaluate patients at 4, 24, 32, and $48 \mathrm{~h}$ and once daily thereafter until 6 days after surgery and assessed at rest. The short form of BPI was assessed at baseline (1 day before surgery), days 2 and 6 post-operatively. The overall satisfaction of the patients who received postoperative analgesia was evaluated before discharge using a 5 -point scale $(1=$ very unsatisfied, 5 = very satisfied).

Assuming an equivalence margin of 10 and a standard deviation of 18 [11], 104 patients were required to achieve the power of $80 \%$ by the non-inferiority test with a one-sided $97.5 \%$ confidence interval (CI), which was equivalent to a two-sided 95\% CI. Considering a 5\% dropout rate, a total sample size of 110 (55 per arm) patients were planned to be randomly assigned to the treatment groups at a 1:1 ratio.

The efficacy outcomes were analyzed with intent-to-treat (ITT) population who were randomized patients receiving at least one dose of the study drug. The AUC of VAS from baseline to $48 \mathrm{~h}$ after surgery compared between study groups was the primary efficacy outcome. The prespecified criteria for non-inferiority analysis were that the upper boundary of the CI of the difference in the AUC of VAS could not be greater than 10 . Non-inferiority was tested using two one-sided $t$ tests [15] on the AUC of VAS against the upper equivalence margin at the $\alpha=0.025$ significance level. If non-inferiority on primary efficacy outcome was met, the superiority analysis was subsequently performed to examine the difference in the AUC of VAS between two treatment groups. The same analysis procedure was applied to the AUC of VAS scores from baseline to $24 \mathrm{~h}$ and to 6 days after surgery.
Secondary endpoints were VAS scores throughout 7 days after surgery (from day 0 to day 6), consumption of rescue medication, BPI pain intensity, BPI interference, post-operative length of stay, and satisfaction. We tested the superiority of the ERDS over the PCA group in ITT population with a two-sided $t$ test or Fisher's exact test on secondary efficacy outcomes. Fisher's exact test was used to test demographics and baseline characteristics. $P<0.05$ was considered statistically significant. The statistical software SAS ${ }^{\circledR}$ version 9.4 (SAS Institute, Cary, NC, USA) was used for statistical analysis.

\section{RESULTS}

Of the 112 screened patients, 110 were randomly assigned to receive ERDS injection or fentanyl by intravenous PCA with equal ratio; two patients withdrew from the study owing to surgery type changed after signing the informed consent; one patient in the PCA group withdrew as premature interruption for personal reasons. A total of 107 patients completed the study: 55 in the ERDS group and 52 in the PCA group (Fig. 1). Demographics and baseline characteristics were summarized in Table 1 . The characteristics of the two treatment groups were balanced at baseline. No statistical differences were found in gender, age, height, weight, BMI, duration of surgery, stage of cancer, surgery type, length of surgical wound, and location of incision.

The AUC of VAS from baseline to $48 \mathrm{~h}$ after surgery were 118.6 (97.5\% CI 95.6-141.6) [LS mean $(97.5 \% \mathrm{CI})]$ in the ERDS group and 176.13 (97.5\% CI 150.8-201.4) in the PCA group. With a prespecified defined margin, the results indicated that the non-inferiority of ERDS to PCA with fentanyl was statistically significant $(P<0.001)$, because the upper limit of the 97.5\% CIs of ERDS was lower than the lower limit of PCA with fentanyl (Table 2). A superiority test and statistical significance were in favor of the ERDS group $(P<0.001)$.

The AUC of VAS from baseline to $24 \mathrm{~h}$ after surgery were $65.1(97.5 \%$ CI $51.0-79.3)$ in the ERDS group and 100.0 (97.5\% CI 85.4-114.7) in the PCA group. The AUC of VAS from baseline 


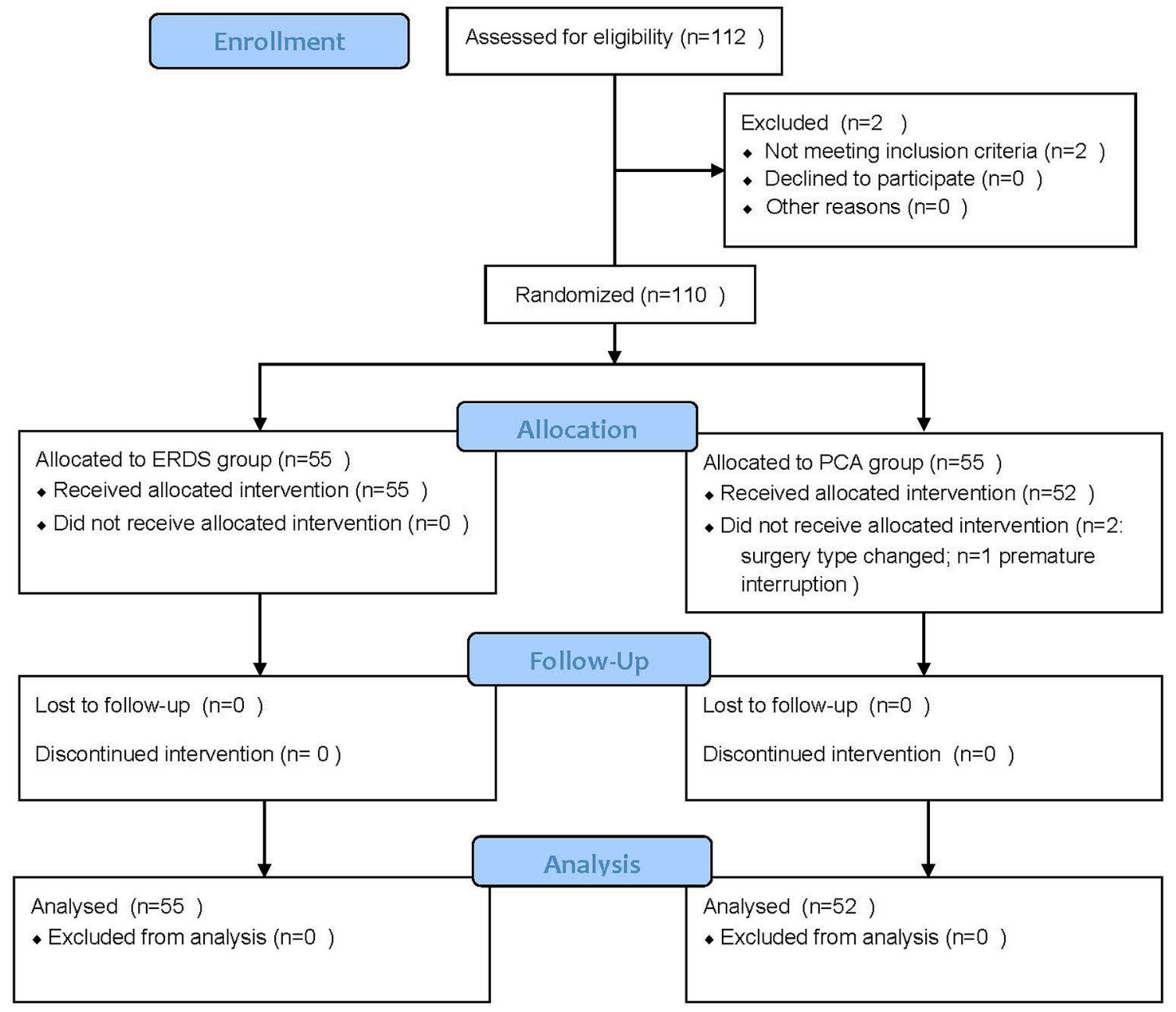

Fig. 1 The CONSORT flow diagram for the study. ERDS, extended-release dinalbuphine sebacate; PCA, patientcontrolled analgesia with fentanyl

to 6 days after surgery were $243.6(97.5 \%$ CI 195.3-292.0) in the ERDS group and 365.9 (97.5\% CI 310.0-421.7) in the PCA group. The AUC of VAS from baseline to $24 \mathrm{~h}$ and 6 days after surgery were also statistically superior to the ERDS group (both $P<0.001$ ).

On superiority analysis for secondary outcomes, post-operative VAS scores at 4, 24, 32, 72,120 , and $144 \mathrm{~h}$ were statistically superior in the ERDS group compared with the PCA group $(P=0.005, \quad P=0.001, \quad P=0.025, \quad P=0.042$, $P=0.011, P<0.001$, respectively; Fig. 2). There was also a trend toward significance in the reduction of VAS scores at 48 and $96 \mathrm{~h}$ after surgery for the ERDS group $(P=0.062$ and $P=0.056$, respectively).
Consumption of morphine dose was $5.90 \pm 3.96$ (mean \pm SD) $\mathrm{mg}$ in the ERDS group and $7.56 \pm 3.28 \mathrm{mg}$ in the PCA group $(P=0.261)$, whereas the mean total of ketorolac use was $57.45 \pm 40.56 \mathrm{mg}$ in the ERDS group and $63.57 \pm 50.66 \mathrm{mg}$ in the PCA group $(P=0.509)$. The number of patients that used morphine after surgery was 30 in the ERDS and nine in the PCA group, whereas those that used ketorolac was 47 in the ERDS group and 23 in the PCA group. Six (11\%) patients in the ERDS group had never used any supplemental analgesics after surgery. Meanwhile, the total amount of fentanyl consumption via PCA was $418.65 \pm 195.37 \mu \mathrm{g}$ in the PCA group. 
Table 1 Summary of the demographic information and baseline characteristics

\begin{tabular}{|c|c|c|}
\hline & $\begin{array}{l}\text { ERDS } \\
(n=55)\end{array}$ & $\begin{array}{l}\text { PCA } \\
(n=52)\end{array}$ \\
\hline \multicolumn{3}{|l|}{ Gender } \\
\hline Male, $n(\%)$ & $37(67.3)$ & $32(61.5)$ \\
\hline Female, $n(\%)$ & $18(32.7)$ & $20(38.5)$ \\
\hline Age (yeasr), mean (SD) & $63.6(9.8)$ & $64.2(8.1)$ \\
\hline Weight $(\mathrm{kg})$, mean $(\mathrm{SD})$ & $63.1(9.2)$ & $\begin{array}{l}63.5 \\
(10.8)\end{array}$ \\
\hline Height $(\mathrm{cm})$, mean $(\mathrm{SD})$ & $\begin{array}{l}161.5 \\
(15.5)\end{array}$ & $\begin{array}{r}162.4 \\
(8.3)\end{array}$ \\
\hline BMI $\left(\mathrm{kg} / \mathrm{m}^{2}\right)$, mean $(\mathrm{SD})$ & $24.2(5.0)$ & $24.0(2.8)$ \\
\hline $\begin{array}{l}\text { Duration of surgery (min), } \\
\text { mean (SD) }\end{array}$ & $224(65)$ & $217(67)$ \\
\hline \multicolumn{3}{|l|}{$\begin{array}{l}\text { Stage of colorectal cancer, } \\
n(\%)\end{array}$} \\
\hline I & $9(16.4)$ & $8(15.4)$ \\
\hline II & $6(10.9)$ & $0(0)$ \\
\hline III & $24(43.6)$ & $28(53.8)$ \\
\hline $\mathrm{V}$ & $8(14.5)$ & $8(15.4)$ \\
\hline Other diseases & $8(14.5)$ & $8(15.4)$ \\
\hline $\begin{array}{l}\text { Length of surgical wound }(\mathrm{cm}) \text {, } \\
\text { mean }(\mathrm{SD})\end{array}$ & $9.5(1.8)$ & $9.7(1.7)$ \\
\hline \multicolumn{3}{|l|}{ Location of incision, $n(\%)$} \\
\hline Left paramedian & $11(20.0)$ & $7(13.5)$ \\
\hline Midline & $30(54.5)$ & $28(53.8)$ \\
\hline Right paramedian & $13(23.6)$ & $16(30.8)$ \\
\hline Others & $1(1.8)$ & $1(1.9)$ \\
\hline \multicolumn{3}{|l|}{ Surgery type, $n$ (\%) } \\
\hline Anterior resection & $21(38.2)$ & $19(36.5)$ \\
\hline Low anterior resection & $14(25.5)$ & $11(21.2)$ \\
\hline Right hemicolectomy & $13(23.6)$ & $16(30.8)$ \\
\hline Left hemicolectomy & $5(9.1)$ & $3(5.8)$ \\
\hline
\end{tabular}

Table 1 continued

\begin{tabular}{lll}
\hline & $\begin{array}{l}\text { ERDS } \\
(\boldsymbol{n}=55)\end{array}$ & $\begin{array}{l}\text { PCA } \\
(\boldsymbol{n}=52)\end{array}$ \\
\hline Others & $2(3.6)$ & $3(5.8)$ \\
\hline
\end{tabular}

Data are presented as mean (SD) or $n(\%)$

$B M I$ body mass index, ERDS extended-release dinalbuphine sebacate, PCA patient-controlled analgesia with fentanyl

Table 2 The area under the curve of visual analogue scale

\begin{tabular}{llcl}
\hline $\begin{array}{l}\text { AUC of } \\
\text { VAS LS } \\
\text { mean } \\
(\mathbf{9 7 . 5 \%}\end{array}$ & $\begin{array}{l}\text { ERDS } \\
(\boldsymbol{n}=\mathbf{5 5})\end{array}$ & PCA $(\boldsymbol{n}=\mathbf{5 2})$ & $\boldsymbol{P}$ value $^{\dagger}$ \\
$\mathbf{C I})$ & & & \\
\hline Baseline to & 65.1 & 100.0 & $<0.001$ \\
$24 \mathrm{~h}$ & $(51.0-79.3)$ & $(85.4-114.7)$ & \\
Baseline to & 118.6 & 176.1 & $<0.001$ \\
48 h & $(95.6-141.6)$ & $(150.8-201.4)$ & \\
Baseline to & 243.6 & 365.9 & $<0.001$ \\
day 6 & $(195.3-292)$ & $(310.0-421.7)$ & \\
\hline
\end{tabular}

Data are presented as least squares mean $(97.5 \%$ confidence interval: lower limit to upper limit)

$E R D S$ extended-release dinalbuphine sebacate, $P C A$ patient-controlled analgesia with fentanyl, $L S$ least squares, $C I$ confidence interval

${ }^{\dagger} P$ value from one-sided $t$ test. $P<0.05$ was considered statistically significant

BPI contained two major parts of assessment: pain intensity and interference in daily activities. Patients were assessed at baseline (before surgery) and at days 2 and 6 after surgery; the results are presented in Table 3. At baseline, no differences were found in the mean pain intensity and interference scores between the two groups. The mean pain intensity score indicated significantly less pain intensity in the ERDS group at days 2 and 6 compared with the PCA group $(2.23 \pm 1.50$ vs. $2.82 \pm 1.44$, 


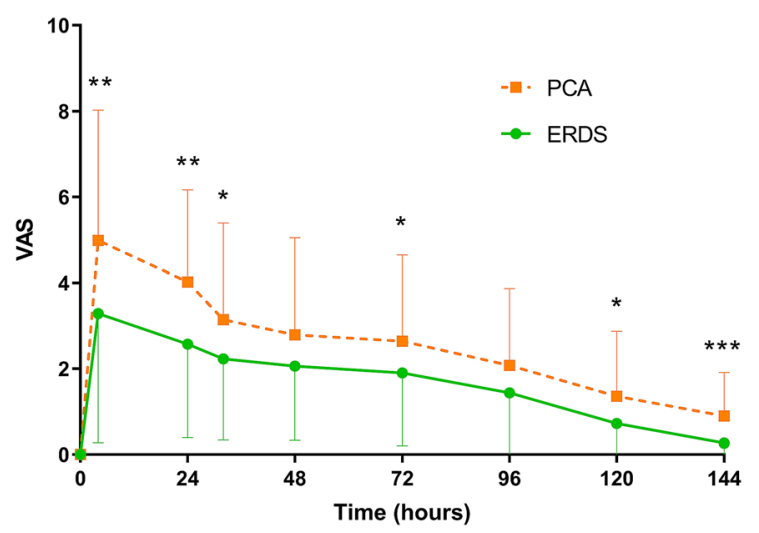

Fig. 2 Post-operative pain intensity. Patients receiving extended-release dinalbuphine sebacate (ERDS) or patientcontrolled analgesia (PCA) with fentanyl were assessed for pain intensity using the visual analogue scale (VAS) at 4, $24,32,48,72,96,120$, and $144 \mathrm{~h}$ post-operatively [intention-to-treat population, mean \pm standard deviation (SD)]. ${ }^{*} P<0.05,{ }^{* *} P<0.01,{ }^{* * *} P<0.001$ vs. PCA group

Table 3 The brief pain inventory following laparotomy

\begin{tabular}{llll}
\hline Mean (SD) & $\begin{array}{l}\text { ERDS } \\
(\boldsymbol{n}=55)\end{array}$ & $\begin{array}{l}\text { PCA } \\
(\boldsymbol{n}=52)\end{array}$ & P value \\
\hline BPI-pain intensity & & \\
Baseline & $0.02(0.24)$ & $0(0.00)$ & 0.322 \\
Day 2 & $2.23(1.50)$ & $2.82(1.44)$ & $0.042^{*}$ \\
Day 6 & $0.73(0.92)$ & $1.16(1.17)$ & $0.034^{*}$ \\
BPI- & & & \\
interference & & & \\
Baseline & $0(0)$ & $0(0)$ & NA \\
Day 2 & $2.50(2.69)$ & $3.29(2.86)$ & 0.141 \\
Day 6 & $0.49(1.07)$ & $1.34(1.78)$ & $0.003^{* *}$ \\
\hline
\end{tabular}

Presented as mean (standard deviation)

ERDS extended-release dinalbuphine sebacate, PCA patient-controlled analgesia with fentanyl, $S D$ standard deviation, $N A$ not available

${ }^{\dagger} P$ value from two-sided $t$ test. $P<0.05$ was considered statistically significant. ${ }^{*} P<0.05,{ }^{* *} P<0.01$

$P=0.042$, and $0.73 \pm 0.92$ vs. $1.16 \pm 1.17$, $P=0.034$, respectively). In addition, the mean interference score at day 6 was significantly lower in the ERDS group than in the PCA group $(0.49 \pm 1.07$ vs. $1.34 \pm 1.78, P=0.003)$. The patients in the ERDS group also reported that the interference was significantly less at day 6 compared with the PCA group in almost all interference parameters such as general activity $(0.45 \pm 1.17$ vs. $1.17 \pm 1.81, P=0.016)$, mood $(0.29 \pm 0.94$ vs. $0.75 \pm 1.36, P=0.043)$, walking $(0.4 \pm 1.01$ vs. $1.08 \pm 1.94, P=0.024)$, work $(1.02 \pm 2.63$ vs. $2.71 \pm 3.81, P=0.008)$, social relationships $\quad(0.25 \pm 0.91 \quad$ vs. $1.12 \pm 2.08, P=0.006)$, and enjoyment of life $(0.35 \pm 1.11$ vs. $1.19 \pm 2.24, P=0.014)$. There was also a trend toward significance in improvement of sleep in the ERDS group $(0.65 \pm 1.59$ vs. $1.37 \pm 2.48, P=0.079)$.

During the last visit, the patients' satisfaction regarding post-operative analgesia was assessed using a 5-point rating scale. Albeit not statistically significant, a slightly higher number of patients receiving ERDS reported as being "highly satisfied" and "satisfied" compared with those receiving intravenous fentanyl using a PCA (91 vs. $82 \%, P=0.167$ ). There was no significant difference in the length of stay between the ERDS and PCA groups $(7.98 \pm 2.57$ vs. $7.31 \pm 2.34$ days, $P=0.160$ ).

A total of 45 patients reported at least one adverse event during the study, that is, 27 (49.1\%) and 18 (32.7\%) patients in the ERDS and PCA groups, respectively. All adverse events were mild or moderate in severity in both treatment groups. The rates of patients reporting drug-related adverse events were $30.9 \%$ and $25.5 \%$ in the ERDS and PCA groups, respectively.

The most frequent adverse drug reaction was pyrexia in both groups [15 (27.3\%) and 14 (25.5\%) patients in the ERDS and PCA groups, respectively, Table 4]. All patients had mild pyrexia. There were 11 and five patients in the ERDS and PCA groups, respectively, who recovered from pyrexia within 1 day. Injection site evaluation in the ERDS group revealed mild erythema in only one patient (1.8\%), who recovered at discharge. Two patients (3.6\%) in the ERDS group reported abnormal hepatic function with alkaline and aspartate transaminase levels above the upper normal limit at 7 days after surgery. The patients returned for 
Table 4 Incidence of adverse drug reactions

\begin{tabular}{lll}
\hline Adverse drug reaction & ERDS & PCA \\
& $(n=55)$ & $(n=52)$ \\
\hline
\end{tabular}

General and administration-site reactions

$\begin{array}{lll}\text { Injection site erythema } & 1(1.8 \%) & 0 \\ \text { Pyrexia } & 15(27.3 \%) & 14(25.5 \%)\end{array}$

Hepatobiliary disorders

Abnormal hepatic $\quad 2(3.6 \%)$
function $^{\mathrm{a}}$

Nervous system disorders

Dizziness $\quad 1(1.8 \%) \quad 0$

Data are presented as $n(\%)$

ERDS extended-release dinalbuphine sebacate, PCA patient-controlled analgesia with fentanyl

a Alkaline and aspartate transaminase levels above the upper normal limit

follow-up 1 week after discharge and recovered. One patient $(1.8 \%)$ experienced mild dizziness 2 days after ERDS administration and recovered within 1 day. There were no adverse events that led to study discontinuation. In addition, no serious adverse events or death occurred during the study period.

\section{DISCUSSION}

In this open-label, randomized controlled trial that assessed the analgesic effect of the extended-release dinalbuphine sebacate after its preemptive administration in open laparotomy by comparing with patients receiving IV PCA with fentanyl, we found that patients who received a single intramuscular injection of ERDS before surgery benefited from ERDS, which resulted in significant reductions in the VAS pain score, $\mathrm{AUC}_{0-24 \mathrm{~h}}$ of VAS, $\mathrm{AUC}_{0-48 \mathrm{~h}}$ of VAS, $\mathrm{AUC}_{0-6}$ days of VAS, and the BPI pain intensity, compared with IV PCA with fentanyl. There was no significant difference in post-operative analgesics between the two treatment groups. More than $90 \%$ of the patients in this study felt satisfied after receiving ERDS, but between- group differences did not reach statistical difference. In addition, ERDS administration resulted in a significantly superior functional improvement in patients, such as in general daily activities, mood, walking, work, social relation, and enjoyment of life.

Many analgesic approaches have been studied and contributed effective outcomes and tolerable safety profile, but few studies reported their therapeutic effects on quality of life. Our findings indicate a significant improvement in post-operative pain intensity and quality of life. It is important to note that improved patient functioning and health-related quality of life were observed in the ERDS group in nearly all BPI interference measures. These results conflicted with other analgesic strategies regarding the benefits of pain scores and post-operative opioids use. One randomized controlled trial stated improved pain scores 2 and $4 \mathrm{~h}$ after surgery in patients undergoing laparotomy in IV PCA with morphine. However, no significant difference was found between groups after $4 \mathrm{~h}$ [16]. With respect to concerns for opioid antagonism, our evaluation regarding the amount of perioperative anesthetics used to determine the potential impact of ERDS on the current standard of care for perioperative anesthesia usage revealed that there was no difference in the anesthetic usage during the induction and surgery between the two groups even though ERDS was administered before the surgery.

To our knowledge, this was the first randomized clinical trial to evaluate the efficacy, safety, and quality of life of ERDS vs. IV PCA with fentanyl in patients undergoing laparotomy who are most in need with long-acting analgesic care. Our study was designed to evaluate an extended-release and strong opioid, expected to assess the analgesic and indirect effects during the recovery period when comparing standard treatment by IV PCA with fentanyl. In this study, we found that the AUC of VAS in ERDS was not only non-inferior to IV PCA but also superior during the overall evaluation period. Clinically meaningful change on pain intensity was affected by surgery type, which determined the severity of pain intensity and recovery period with feeling of pain. In 
general, a change of 10 for the $100 \mathrm{~mm}$ VAS or VAS below 3.3 was considered as significant clinical meaning [17]. In between-group comparison, 17 and $14 \mathrm{~mm}$ of VAS were found at 4 and $24 \mathrm{~h}$ after surgery (Fig. 2). More than $10 \mathrm{~mm}$ VAS reduction from $4 \mathrm{~h}$ after surgery was found at $32 \mathrm{~h}$ after surgery and beyond. The average VAS assessment on all evaluation periods was below 3.3. All analysis results revealed ERDS could effectively ease post-operative pain in the first $24 \mathrm{~h}$ and last long enough to cover a 7-day recovery period, which reflects the promise on patients' quality of life.

There were some limitations to this study. First, the open-label study design caused the differences between pre-emptive and post-operative analgesics might have had some effects on psychological expectation in terms of postoperative pain intensity. Second, the duration of patient-controlled device use was limited. The PCA group utilized intravenous fentanyl administration with the device only during the first $48 \mathrm{~h}$ after surgery and not throughout the entire study period. Supplemental analgesics were necessary to control pain beyond the first $48 \mathrm{~h}$ after surgery. It also reflected on interference of activity on day 6 (Table 3). Third, the study had a small size and was conducted at one study center; therefore, more data were needed to translate similar results on other surgery types.

\section{CONCLUSIONS}

A single dose of ERDS provided better post-operative analgesia and better quality of life than PCA using intravenous fentanyl administered post-operatively. The pre-emptive analgesia used in this study should be considered in patients undergoing open laparotomy because of its efficacy in attenuating post-operative pain and contribution to improved quality of life during the recovery period.

\section{ACKNOWLEDGEMENTS}

The authors thank the participants of this study.
Funding. This study and Rapid Service Fee were funded by the Ministry of Science and Technology (MOST 109-2314-B-037-035, MOST 109-2314-B-037-040, MOST 109-2314-B-037046-MY3), the Ministry of Health and Welfare (MOHW107-TDU-B-212-123006, MOHW107TDU-B-212-114026B, MOHW108-TDU-B-212133006, MOHW109-TDU-B-212-134026), Health and welfare surcharge of tobacco products, Kaohsiung Medical University Hospital (KMUH108-8R34, KMUH108-8R35, KMUH1088M33, KMUH108-8M35, KMUH108-8M36, KMUHS10801, KMUHSA10804, KMUHS10807, KMUH-DK109003, KMUH-DK109005 3, KMUHS10903), and Center for Cancer Research (KMU-TC108A04) and Cohort Research Center Grant (KMU-TC108B07), Kaohsiung Medical University.

Authorship. All named authors meet the International Committee of Medical Journal Editors (ICMJE) criteria for authorship of this article, take responsibility for the integrity of the work as a whole, and have given their approval for this version to be published.

Authorship Contributions. Tsai HL and Wang JY designed and wrote the study protocol; Su MP, Cheng KI, Su WC, Ma CJ, Yeh YS, Chen YC, and Li CC participated recruitment, data acquisition, and outcomes interpretation; Huang CW performed data management and statistical analysis; Chang TK conducted the literature search and draft the manuscript; and Wang JY reviewed critical revision of the manuscript. All authors approved the final version of the manuscript.

Disclosures. Tsung-Kun Chang, Ching-Wen Huang, Wei-Chih Su, Hsiang-Lin Tsai, ChengJen Ma, Yung-Sung Yeh, Yen-Cheng Chen, Ching-Chun Li, Kuang-I Cheng, Miao-Pei Su and Jaw-Yuan Wang have nothing to disclose.

Compliance with Ethics Guidelines. The study was approved by the hospital's research ethics committee (Institutional Review Board of Kaohsiung Medical University Hospital, KMUHIRB-F(I)-20170089) and was registered in the Clinicaltrials.gov (NCT03296488, Principal 
investigator: Wang JY, M.D., Ph.D.). The study protocol was conducted at the Kaohsiung Medical University Hospital from November 2017 to July 2019, in accordance with the Declaration of Helsinki, International Conference on Harmonization of Good Clinical Practice, and local regulatory requirements. All participants signed informed consent before initiation of study assessments.

Data Availability. The datasets generated during and/or analyzed during the current study are available from the corresponding author on reasonable request.

Open Access. This article is licensed under a Creative Commons Attribution-NonCommercial 4.0 International License, which permits any non-commercial use, sharing, adaptation, distribution and reproduction in any medium or format, as long as you give appropriate credit to the original author(s) and the source, provide a link to the Creative Commons licence, and indicate if changes were made. The images or other third party material in this article are included in the article's Creative Commons licence, unless indicated otherwise in a credit line to the material. If material is not included in the article's Creative Commons licence and your intended use is not permitted by statutory regulation or exceeds the permitted use, you will need to obtain permission directly from the copyright holder. To view a copy of this licence, visit http://creativecommons.org/licenses/by$\mathrm{nc} / 4.0 /$.

\section{REFERENCES}

1. Toro MM, John S, Faruqui AR. Pattern of use of analgesics in post-operative pain management in adults undergoing laparotomy surgery: a prospective observational study. Int Surg J. 2018;5(2): 662-7. https://doi.org/10.18203/2349-2902. isj20180372.

2. Bruce J, Krukowski ZH. Quality of life and chronic pain four years after gastrointestinal surgery. Dis Colon Rectum. 2006;49(9):1362-70. https://doi. org/10.1007/s10350-006-0575-5.
3. Gan TJ, Habib AS, Miller TE, White W, Apfelbaum JL. Incidence, patient satisfaction, and perceptions of post-surgical pain: results from a US national survey. Curr Med Res Opin. 2014;30(1):149-60. https://doi.org/10.1185/03007995.2013.860019.

4. Joshi GP, Ogunnaike BO. Consequences of inadequate postoperative pain relief and chronic persistent postoperative pain. Anesthesiol Clin N Am. 2005;23(1):21-36. https://doi.org/10.1016/j.atc. 2004.11.013.

5. Gan TJ. Poorly controlled postoperative pain: prevalence, consequences, and prevention. J Pain Res. 2017;10:2287-98. https://doi.org/10.2147/JPR. S144066.

6. Son HJ, Kim SH, Ryu JO, et al. Device-related error in patient-controlled analgesia: analysis of 82,698 patients in a tertiary hospital. Anesth Analg. 2019;129(3):720-5. https://doi.org/10.1213/ANE. 0000000000003397.

7. Niiyama Y, Matsuoka N. Efficacy of intravenous patient-controlled analgesia (IV-PCA) using fentanyl compared with IV-PCA using morphine after abdominal surgery: a prospective randomized study. J Anesth Clin Res. 2016;7(1):598. https://doi. org/10.4172/2155-6148.1000598.

8. Imani F, Faiz HR, Sedaghat M, Hajiashrafi M. Effects of adding ketamine to fentanyl plus acetaminophen on postoperative pain by patient-controlled analgesia in abdominal surgery. Anesthesiol Pain Med. 2013;4(1):e12162. https://doi.org/10. 5812/aapm.12162.

9. El Sayed MH, Mokbel EM. Postoperative analgesia after major abdominal surgery: Fentanyl-bupivacaine patient-controlled epidural analgesia versus fentanyl patient-controlled intravenous analgesia. Egypt J Anaesth. 2014;30(4):393-7. https://doi.org/ 10.1016/j.egja.2014.06.002.

10. Tien YE, Huang WC, Kuo HY, et al. Pharmacokinetics of dinalbuphine sebacate and nalbuphine in human after intramuscular injection of dinalbuphine sebacate in an extended-release formulation. Biopharm Drug Dispos. 2017;38(8):494-7. https://doi.org/10.1002/bdd.2088.

11. Yeh CY, Jao SW, Chen JS, et al. Sebacoyl dinalbuphine ester extended-release injection for longacting analgesia: a multicenter, randomized, double-blind, and placebo-controlled study in hemorrhoidectomy patients. Clin J Pain. 2017;33(5): 429-34. 0000000000000417.

12. Yeh YC, Lin TF, Lin FS, Wang YP, Lin CJ, Sun WZ. Combination of opioid agonist and agonist-antagonist: patient-controlled analgesia requirement and 
adverse events among different-ratio morphine and nalbuphine admixtures for postoperative pain. $\mathrm{Br} \mathrm{J}$ Anaesth. 2008;101(4):542-8. https://doi.org/10. 1093/bja/aen213.

13. Chen MK, Chau SW, Shen YC, et al. Dose-dependent attenuation of intravenous nalbuphine on epidural morphine-induced pruritus and analgesia after cesarean delivery. Kaohsiung J Med Sci. 2014;30(5):248-53. https://doi.org/10.1016/j.kjms. 2014.01.001.

14. Huang CC, Sun WZ, Wong CS. Prevention of chronic postsurgical pain: the effect of preventive and multimodal analgesia. Asian J Anesthesiol. 2018;56(3):74-82. 201809_56(3).0002.
15. Schuirmann DJ. A comparison of the two one-sided tests procedure and the power approach for assessing the equivalence of average bioavailability. J Pharmacokinet Biopharm. 1987;15(6):657-80. https://doi.org/10.1007/BF01068419.

16. Yaghoubi S, Pourfallah R, Barikani A, Kayalha H. The postoperative analgesic effect of morphine and paracetamol in the patients undergoing laparotomy, using PCA method. Glob J Health Sci. 2014;6(1): 207-14. https://doi.org/10.5539/gjhs.v6n1p207.

17. Myles PS, Myles DB, Galagher W, et al. Measuring acute postoperative pain using the visual analog scale: the minimal clinically important difference and patient acceptable symptom state. Br J Anaesth. 2017;118(3): 424-9. https://doi.org/10.1093/bja/aew466. 